Proyecciones Journal of Mathematics

Vol. 32, No 3, pp. 199-213, September 2013.

Universidad Católica del Norte

Antofagasta - Chile

\title{
Difference inequalities of fractional order
}

\author{
J. Jagan Mohan \\ BITS Pilani, India \\ and \\ G. V.S.R. Deekshitulu \\ JNTU Kakinada, India \\ Received: December 2011. Accepted : May 2013
}

\begin{abstract}
In this paper, we establish some new difference inequalities of fractional order which provide explicit bounds on unknown functions and can be used as an effective tool in the development of the theory of fractional order difference equations.
\end{abstract}

Key Words : Finite difference, inequality, fractional order.

AMS Classification : 39A10, 39A99 


\section{Introduction}

Difference equations usually describe the evolution of certain phenomena over the course of time. The theory of difference equations has been developed as a natural discrete analogue of corresponding theory of differential equations. Many physical problems arising in a wide variety of applications are governed by finite difference equations.

The notions of fractional differential equations may be traced back to the works of Euler, but the idea of fractional difference equations is very recent. Diaz and Osler [7] defined the fractional difference by the rather natural approach of allowing the index of differencing, in the standard expression for the $n^{\text {th }}$ difference, to be any real or complex number. Later, Hirota [8] defined the fractional difference using Taylor series. In 2002, Atsushi Nagai [2] introduced another definition of fractional difference which is a slight modification of Hirotas definition. In 2010, G. V. S. R. Deekshitulu and J. Jagan Mohan [3] modified the definition of Atsushi Nagai [2] in such a way that the expression for fractional difference does not involve any difference operator and using which some basic difference inequalities have been established.

The theory of inequalities is always of great importance for the development of many branches of mathematics. This field is dynamic and experiencing an explosive growth in both theory and applications. As a response to the needs of diverse applications, a large variety of inequalities have been proposed and studied in the literature. Since the integral inequalities with explicit estimates are so important in the study of properties of solutions of differential and integral equations, their finite difference (or discrete) analogues should also be useful in the study of properties of solutions of finite difference equations. The finite difference version of the well known Gronwall inequality seems to have appeared first in the work of Mikeladze in 1935. It is well recognized that the discrete version of Gronwalls inequality provides a very useful and important tool in proving convergence of the discrete variable methods. In view of wider applications, finite difference inequalities with explicit estimates have been generalized, extended and used considerably in the development of the theory of finite difference equations.

In the present paper, the authors considered an initial value problem of fractional order and some difference inequalities and comparison results are obtained. 


\section{Notations and Terminology}

Throughout the article, we shall use the following notations and definitions [1].

Let $\mathbf{Z}$ and $\mathbf{R}$ denote the set of all integers and the set of all real numbers respectively and $\mathbf{R}^{+}=[0, \infty)$. $\mathbf{N}=\{0,1,2, \ldots\}$ be the set of all natural numbers including zero and $\mathbf{N}_{a}^{+}=\{a, a+1, a+2, \ldots\}$ where $a \in \mathbf{N}$. Let $u(n): \mathbf{N}_{0}^{+} \rightarrow \mathbf{R}$ then for all $a, b \in \mathbf{N}_{a}^{+}$and $a>b, \sum_{j=a}^{b} u(j)=0$ and $\prod_{j=a}^{b} u(j)=1$, i.e. empty sums and products are taken to be 0 and 1 respectively. If $n-1$ and $n$ are in $\mathbf{N}_{a}^{+}$, then for this function $u(n)$ the backward difference operator $\nabla$ is defined as $\nabla u(n)=u(n)-u(n-1)$. The extended binomial coefficient $\left(\begin{array}{l}a \\ n\end{array}\right),(a \in \mathbf{R}, n \in \mathbf{Z})$ is defined by

$$
\left(\begin{array}{l}
a \\
n
\end{array}\right)= \begin{cases}\frac{\Gamma(a+1)}{\Gamma(a-n+1) \Gamma(n+1)} & \mathrm{n}>0 \\
1 & \mathrm{n}=0 \\
0 & \mathrm{n}<0\end{cases}
$$

G. V. S. R. Deekshitulu and J. Jagan Mohan [6] modified the definition of fractional difference given by Atsushi Nagai [2] for $0<\alpha<1$ as follows.

Definition 2.1. The fractional sum operator of order $\alpha$ is defined as

$$
\nabla^{-\alpha} u(n)=\sum_{j=0}^{n-1}\left(\begin{array}{c}
j+\alpha-1 \\
j
\end{array}\right) u(n-j)=\sum_{j=1}^{n}\left(\begin{array}{c}
n-j+\alpha-1 \\
n-j
\end{array}\right) u(j)
$$

Definition 2.2. The fractional difference operator of order $\alpha$ is defined as

$$
\nabla^{\alpha} u(n)=\nabla^{\alpha-1}[\nabla u(n)]=\sum_{j=0}^{n-1}\left(\begin{array}{c}
j-\alpha \\
j
\end{array}\right) \nabla u(n-j) .
$$

Remark 1. Let $u(n), v(n): \mathbf{N}_{0}^{+} \rightarrow \mathbf{R} ; \alpha, \beta \in \mathbf{R}$ such that $0<\alpha, \beta, \alpha+$ $\beta<1$ and $c, d$ are scalars. Then the fractional order difference operator satisfies the following properties.

1. $\nabla^{\alpha} \nabla^{\beta} u(n)=\nabla^{\alpha+\beta} u(n)$.

2. $\nabla^{\alpha}[c u(n)+d v(n)]=c \nabla^{\alpha} u(n)+d \nabla^{\alpha} v(n)$.

3. $\nabla^{\alpha} \nabla^{-\alpha} u(n)=u(n)$.

4. $\nabla^{-\alpha} \nabla^{\alpha} u(n)=u(n)-u(0)$. 
Definition 2.3. Let $f(n, r)$ be any function defined for $n \in \mathbf{N}_{0}^{+}, 0 \leq r<$ $\infty$. Then a nonlinear difference equation of order $\alpha \in \mathbf{R}, 0<\alpha<1$ together with an initial condition is of the form

$$
\nabla^{\alpha} u(n+1)=f(n, u(n)), u(0)=u_{0} .
$$

Using (2.3), the solution of (2.4) is expressed as a recurrence relation involving the values of the unknown function at the previous arguments as follows.

$u(n)=u_{0}+\sum_{j=0}^{n-1}\left(\begin{array}{c}n-j+\alpha-2 \\ n-j-1\end{array}\right) f(j, u(j))=u_{0}+\sum_{j=0}^{n-1} B(n-1, \alpha ; j) f(j, u(j))$

where $B(n, \alpha ; j)=\left(\begin{array}{c}n-j+\alpha-1 \\ n-j\end{array}\right)$ for $0 \leq j \leq n$. Recently [6], the authors have established the following fractional order discrete Gronwall - Bellman inequality.

Theorem 2.1. Let $u(n), a(n)$ and $b(n)$ be real valued nonnegative functions defined on $\mathbf{N}_{0}^{+}$. For $n \in \mathbf{N}_{0}^{+}$, if

$$
\nabla^{\alpha} u(n+1) \leq a(n) u(n)+b(n)
$$

then

$$
\begin{gathered}
u(n) \leq u(0) \prod_{j=0}^{n-1}[1+B(n-1, \alpha ; j) a(j)]+ \\
\sum_{j=0}^{n-1}\left[B(n-1, \alpha ; j) b(j) \prod_{k=j+1}^{n-1}[1+B(n-1, \alpha ; k) a(k)]\right] .
\end{gathered}
$$

\section{Main Results}

In the present section, we establish some new fractional order difference inequalities.

Theorem 3.1. Let $u(n), a(n), b(n)$ and $c(n)$ are real valued nonnegative functions defined on $\mathbf{N}_{0}^{+}$and $\nabla^{\alpha} c(n) \geq 0$ for $n \in \mathbf{N}_{0}^{+}$. Let $L: \mathbf{N}_{0}^{+} \times \mathbf{R}^{+} \rightarrow$ $\mathbf{R}^{+}$be a function such that

$$
0 \leq L(n, x)-L(n, y) \leq m(n, y)(x-y)
$$


for $n \in \mathbf{N}_{0}^{+}, x \geq y \geq 0$, where $M(n, y)$ is a real valued nonnegative function defined for $n \in \mathbf{N}_{0}^{+}, y \in \mathbf{R}^{+}$. If

$$
u(n) \leq a(n)+b(n)\left(c(n)+\sum_{j=0}^{n-1} B(n-1, \alpha ; j) L(j, u(j))\right)
$$

for $n \in \mathbf{N}_{0}^{+}$, then

$$
\begin{aligned}
u(n) \leq a(n)+b(n)\left(c(0) \prod_{j=0}^{n-1}[1+B(n-1, \alpha ; j) M(j, a(j)) b(j)]\right. \\
+\sum_{j=0}^{n-1} B(n-1, \alpha ; j)\left[\nabla^{\alpha} c(j+1)+L(j, a(j))\right] \\
\left.\quad \prod_{k=j+1}^{n-1}[1+B(n-1, \alpha ; k) M(k, a(k)) b(k)]\right)
\end{aligned}
$$

for $n \in \mathbf{N}_{0}^{+}$.

Proof. Define a function $z(n)$ by

$$
z(n)=c(n)+\sum_{j=0}^{n-1} B(n-1, \alpha ; j) L(j, u(j)) .
$$

Then $z(0)=c(0)$ and (3.2) can be restated as

$$
u(n) \leq a(n)+b(n) z(n) .
$$

From (3.1), (3.4) and (3.5) we have

$$
\begin{aligned}
& \nabla^{\alpha} z(n+1)=\nabla^{\alpha} c(n+1)+L(n, u(n)) \\
& \leq \nabla^{\alpha} c(n+1)+L(n, a(n)+b(n) z(n)) \\
& =\left[\nabla^{\alpha} c(n+1)+L(n, a(n))\right]+[L(n, a(n)+b(n) z(n))-L(n, a(n))] \\
& \leq\left[\nabla^{\alpha} c(n+1)+L(n, a(n))\right]+M(n, a(n))(b(n) z(n)) .
\end{aligned}
$$


Now an application of Theorem 2.1 to (3.6) yields

$$
\begin{gathered}
z(n) \leq c(0) \prod_{j=0}^{n-1}[1+B(n-1, \alpha ; j) M(j, a(j)) b(j)] \\
+\sum_{j=0}^{n-1} B(n-1, \alpha ; j)\left[\nabla^{\alpha} c(j+1)+L(j, a(j))\right] \\
\quad \prod_{k=j+1}^{n-1}[1+B(n-1, \alpha ; k) M(k, a(k)) b(k)] .
\end{gathered}
$$

Using (3.7) in (3.5) we get the required inequality in (3.3).

Theorem 3.2. Let $u(n), a(n), b(n)$ and $c(n)$ are real valued nonnegative functions defined on $\mathbf{N}_{0}^{+}$and $\nabla^{\alpha} c(n) \geq 0$ for $n \in \mathbf{N}_{0}^{+}$. Let $L: \mathbf{N}_{0}^{+} \times \mathbf{R}^{+} \rightarrow$ $\mathbf{R}^{+}$be a function such that

$$
0 \leq L(n, x)-L(n, y) \leq m(n, y) \phi^{-1}(x-y)
$$

for $n \in \mathbf{N}_{0}^{+}, x \geq y \geq 0$, where $M(n, y)$ is a real valued nonnegative function defined for $n \in \mathbf{N}_{0}^{+}, y \in \mathbf{R}^{+}$and $\phi: \mathbf{R}^{+} \rightarrow \mathbf{R}^{+}$is a continuous and strictly increasing function with $\phi(0)=0, \phi^{-1}$ is the inverse of $\phi$ and

$$
\phi^{-1}(x y) \leq \phi^{-1}(x) \phi^{-1}(y)
$$

for $x, y \in \mathbf{R}^{+}$. If

$$
u(n) \leq a(n)+b(n) \phi\left(c(n)+\sum_{j=0}^{n-1} B(n-1, \alpha ; j) L(j, u(j))\right)
$$

for $n \in \mathbf{N}_{0}^{+}$, then

$$
\begin{aligned}
u(n) \leq a(n) & +b(n) \phi\left(c(0) \prod_{j=0}^{n-1}\left[1+B(n-1, \alpha ; j) M(j, a(j)) \phi^{-1}(b(j))\right]\right. \\
& +\sum_{j=0}^{n-1} B(n-1, \alpha ; j)\left[\nabla^{\alpha} c(j+1)+L(j, a(j))\right] \\
& \left.\prod_{k=j+1}^{n-1}\left[1+B(n-1, \alpha ; k) M(k, a(k)) \phi^{-1}(b(k))\right]\right)
\end{aligned}
$$

for $n \in \mathbf{N}_{0}^{+}$. 
Proof. Define a function $z(n)$ by

$$
z(n)=c(n)+\sum_{j=0}^{n-1} B(n-1, \alpha ; j) L(j, u(j)) .
$$

Then $z(0)=c(0)$ and $(3.10)$ can be restated as

$$
u(n) \leq a(n)+b(n) \phi(z(n)) .
$$

From (3.8), (3.9), (3.12) and (3.13) we have

$$
\begin{aligned}
\nabla^{\alpha} u(n+1) & =\nabla^{\alpha} c(n+1)+L(n, u(n)) \\
& \leq \nabla^{\alpha} c(n+1)+L(n, a(n)+b(n) \phi(z(n))) \\
& =\left[\nabla^{\alpha} c(n+1)+L(n, a(n))\right]+[L(n, a(n)+b(n) \phi(z(n)))-L(n, a(n))] \\
& \leq\left[\nabla^{\alpha} c(n+1)+L(n, a(n))\right]+M(n, a(n)) \phi^{-1}(b(n) \phi(z(n))) \\
& \leq\left[\nabla^{\alpha} c(n+1)+L(n, a(n))\right]+M(n, a(n)) \phi^{-1}(b(n)) z(n) .
\end{aligned}
$$

Now an application of Theorem 2.1 to (3.14) yields

$$
\begin{aligned}
z(n) & \leq c(0) \prod_{j=0}^{n-1}\left[1+B(n-1, \alpha ; j) M(j, a(j)) \phi^{-1}(b(j))\right] \\
& +\sum_{j=0}^{n-1} B(n-1, \alpha ; j)\left[\nabla^{\alpha} c(j+1)+L(j, a(j))\right] \\
& \prod_{k=j+1}^{n-1}\left[1+B(n-1, \alpha ; k) M(k, a(k)) \phi^{-1}(b(k))\right] .
\end{aligned}
$$

Using (3.15) in (3.13) we get (3.11).

Theorem 3.3. Let $u(n), a(n), b(n), g(n)$ and $h(n)$ are real valued nonnegative functions defined on $\mathbf{N}_{0}^{+}$and $p>1$ be a real constant. If

$$
u^{p}(n) \leq a(n)+b(n) \sum_{j=0}^{n-1} B(n-1, \alpha ; j)\left[g(j) u^{p}(j)+h(j) u(j)\right]
$$


for $n \in \mathbf{N}_{0}^{+}$, then

$$
\begin{aligned}
& u(n) \leq\left\{a(n)+b(n) \sum_{j=0}^{n-1} B(n-1, \alpha ; j)\left(g(j) a(j)+h(j)\left(\frac{p-1}{p}+\frac{a(j)}{p}\right)\right)\right. \\
& \left.(3.17) \times \prod_{k=j+1}^{n-1}\left[1+B(n-1, \alpha ; k) b(k)\left(g(k)+\frac{h(k)}{p}\right)\right]\right\}^{\frac{1}{p}}
\end{aligned}
$$

for $n \in \mathbf{N}_{0}^{+}$.

Proof. Define a function $z(n)$ by

$$
z(n)=\sum_{j=0}^{n-1} B(n-1, \alpha ; j)\left[g(j) u^{p}(j)+h(j) u(j)\right] .
$$

Then $z(0)=0$,

$$
\nabla^{\alpha} z(n+1)=g(n) u^{p}(n)+h(n) u(n)
$$

and (3.16) can be written as

$$
u^{p}(n) \leq a(n)+b(n) z(n) .
$$

From (3.19) and using the elementary inequality

$$
x^{\frac{1}{p}} y^{\frac{1}{q}} \leq \frac{x}{p}+\frac{y}{q}
$$

where $x \geq 0, y \geq 0$ and $\frac{1}{p}+\frac{1}{q}=1$, we observe that

$(3.22) u(n) \leq(a(n)+b(n) z(n))^{\frac{1}{p}}(1)^{\frac{1}{\left(\frac{p}{p-1}\right)}} \leq \frac{p-1}{p}+\frac{a(n)}{p}+\frac{b(n)}{p} z(n)$.

From (3.19) and using (3.20) and (3.22), we get

$$
\begin{aligned}
\nabla^{\alpha} z(n+1) & \leq g(n)[a(n)+b(n) z(n)]+h(n)\left[\frac{p-1}{p}+\frac{a(n)}{p}+\frac{b(n)}{p} z(n)\right] \\
(3.23) & =b(n)\left[g(n)+\frac{h(n)}{p}\right] z(n)+\left[g(n) a(n)+h(n)\left(\frac{p-1}{p}+\frac{a(n)}{p}\right)\right] .
\end{aligned}
$$

Now an application of Theorem 2.1 to (3.23) yields 


$$
\begin{array}{r}
\mathrm{z}(\mathrm{n}) \leq \sum_{j=0}^{n-1} B(n-1, \alpha ; j)\left(g(j) a(j)+h(j)\left(\frac{p-1}{p}+\frac{a(j)}{p}\right)\right) \\
\prod_{k=j+1}^{n-1}\left[1+B(n-1, \alpha ; k) b(k)\left(g(k)+\frac{h(k)}{p}\right)\right] .
\end{array}
$$

Using (3.24) in (3.20) we get the required inequality in (3.17).

Theorem 3.4. Let $u(n), b(n), g(n)$ and $h(n)$ are real valued nonnegative functions defined on $\mathbf{N}_{0}^{+}$and $c(n)$ be a real valued positive and nondecreasing function defined on $\mathbf{N}_{0}^{+}$. Let $p>1$ be a real constant. If

$$
u^{p}(n) \leq c^{p}(n)+b(n) \sum_{j=0}^{n-1} B(n-1, \alpha ; j)\left[g(j) u^{p}(j)+h(j) u(j)\right]
$$

for $n \in \mathbf{N}_{0}^{+}$, then

$$
\begin{aligned}
u(n) \leq & c(n)\left\{1+b(n) \sum_{j=0}^{n-1} B(n-1, \alpha ; j)\left(g(j)+h(j) c^{1-p}(j)\right)\right. \\
& \left.\times \prod_{k=j+1}^{n-1}\left[1+B(n-1, \alpha ; k) b(k)\left(g(k)+\frac{h(k)}{p} c^{1-p}(k)\right)\right]\right\}^{\frac{1}{p}}
\end{aligned}
$$

for $n \in \mathbf{N}_{0}^{+}$.

Proof. Since $c(n)$ is positive and nondecreasing function for $n \in \mathbf{N}_{0}^{+}$, from (3.25) we observe that

$$
\left(\frac{u(n)}{c(n)}\right)^{p} \leq 1+b(n) \sum_{j=0}^{n-1} B(n-1, \alpha ; j)\left[g(j)\left(\frac{u(j)}{c(j)}\right)^{p}+h(j) c^{1-p}(j)\left(\frac{u(j)}{c(j)}\right)^{p}\right] .
$$

Now an application of Theorem 3.3 to (3.27) yields the desired inequality in (3.26).

Theorem 3.5. Let $u(n), a(n)$ and $b(n)$ are real valued nonnegative functions defined on $\mathbf{N}_{0}^{+}$and $p>1$ be a real constant. Let $L: \mathbf{N}_{0}^{+} \times \mathbf{R}^{+} \rightarrow \mathbf{R}^{+}$ be a function such that

$$
0 \leq L(n, x)-L(n, y) \leq m(n, y)(x-y)
$$


for $n \in \mathbf{N}_{0}^{+}, x \geq y \geq 0$, where $M(n, y)$ is a real valued nonnegative function defined for $n \in \mathbf{N}_{0}^{+}, y \in \mathbf{R}^{+}$. If

$$
u^{p}(n) \leq a(n)+b(n) \sum_{j=0}^{n-1} B(n-1, \alpha ; j) L(j, u(j))
$$

for $n \in \mathbf{N}_{0}^{+}$, then

$$
\begin{aligned}
u(n) \leq & \left\{a(n)+b(n) \sum_{j=0}^{n-1} B(n-1, \alpha ; j) L\left(j, \frac{p-1}{p}+\frac{a(j)}{p}\right)\right. \\
& \left.\times \prod_{k=j+1}^{n-1}\left[1+B(n-1, \alpha ; k) M\left(k, \frac{p-1}{p}+\frac{a(k)}{p}\right) \frac{b(k)}{p}\right]\right\}^{\frac{1}{p}}
\end{aligned}
$$

for $n \in \mathbf{N}_{0}^{+}$.

Proof. Define a function $z(n)$ by

$$
z(n)=\sum_{j=0}^{n-1} B(n-1, \alpha ; j) L(j, u(j)) .
$$

Then $z(0)=0$ and (3.29) can be restated as

$$
u^{p}(n) \leq a(n)+b(n) z(n) .
$$

From (3.32) and using the elementary inequality

$$
x^{\frac{1}{p}} y^{\frac{1}{q}} \leq \frac{x}{p}+\frac{y}{q}
$$

where $x \geq 0, y \geq 0$ and $\frac{1}{p}+\frac{1}{q}=1$, we observe that

$$
u(n) \leq(a(n)+b(n) z(n))^{\frac{1}{p}}(1)^{\frac{1}{\left(\frac{p}{p-1}\right)}} \leq \frac{p-1}{p}+\frac{a(n)}{p}+\frac{b(n)}{p} z(n) .
$$

From (3.31) and using (3.32) and (3.34), we get

$$
\nabla^{\alpha} z(n+1)=L(n, u(n))
$$




$$
\begin{gathered}
\leq L\left(n, \frac{p-1}{p}+\frac{a(n)}{p}+\frac{b(n)}{p} z(n)\right) \\
=L\left(n, \frac{p-1}{p}+\frac{a(n)}{p}\right)+\left[L\left(n, \frac{p-1}{p}+\frac{a(n)}{p}+\frac{b(n)}{p} z(n)\right)\right. \\
\left.-L\left(n, \frac{p-1}{p}+\frac{a(n)}{p}\right)\right] \\
\leq L\left(n, \frac{p-1}{p}+\frac{a(n)}{p}\right)+M\left(n, \frac{p-1}{p}+\frac{a(n)}{p}\right)\left(\frac{b(n)}{p} z(n)\right) .
\end{gathered}
$$

Now an application of Theorem 2.1 to (3.35) yields

$$
\begin{gathered}
z(n) \leq \sum_{j=0}^{n-1} B(n-1, \alpha ; j) L\left(j, \frac{p-1}{p}+\frac{a(j)}{p}\right) \\
\prod_{k=j+1}^{n-1}\left[1+B(n-1, \alpha ; k) M\left(k, \frac{p-1}{p}+\frac{a(k)}{p}\right) \frac{b(k)}{p}\right] .
\end{gathered}
$$

Using (3.36) in (3.32) we get the required inequality in (3.30).

Theorem 3.6. Let $u(n), a(n)$ and $b(n)$ are real valued nonnegative functions defined on $\mathbf{N}_{0}^{+}$and $p>1$ be a real constant.

Let $L: \mathbf{N}_{0}^{+} \times \mathbf{R}^{+} \rightarrow \mathbf{R}^{+}$be a function such that

$$
0 \leq L(n, x)-L(n, y) \leq m(n, y) \phi^{-1}(x-y)
$$

for $n \in \mathbf{N}_{0}^{+}, x \geq y \geq 0$, where $M(n, y)$ is a real valued nonnegative function defined for $n \in \mathbf{N}_{0}^{+}, y \in \mathbf{R}^{+}$and $\phi: \mathbf{R}^{+} \rightarrow \mathbf{R}^{+}$is a continuous and strictly increasing function with $\phi(0)=0, \phi^{-1}$ is the inverse of $\phi$ and

$$
\phi^{-1}(x y) \leq \phi^{-1}(x) \phi^{-1}(y)
$$

for $x, y \in \mathbf{R}^{+}$. if

$$
u^{p}(n) \leq a(n)+b(n) \phi\left(\sum_{j=0}^{n-1} B(n-1, \alpha ; j) L(j, u(j))\right)
$$


for $n \in \mathbf{N}_{0}^{+}$, then

$u(n) \leq\left\{a(n)+b(n) \phi\left(\sum_{j=0}^{n-1} B(n-1, \alpha ; j) L\left(j, \frac{p-1}{p}+\frac{a(j)}{p}\right)\right.\right.$

$$
\left.\left.\times \prod_{k=j+1}^{n-1}\left[1+B(n-1, \alpha ; k) M\left(k, \frac{p-1}{p}+\frac{a(k)}{p}\right) \phi^{-1}\left(\frac{b(k)}{p}\right)\right]\right)\right\}^{\frac{1}{p}}
$$

for $n \in \mathbf{N}_{0}^{+}$.

Proof. Define a function $z(n)$ by

$$
z(n)=\sum_{j=0}^{n-1} B(n-1, \alpha ; j) L(j, u(j))
$$

Then $z(0)=0$ and $(3.39)$ can be restated as

$$
u^{p}(n) \leq a(n)+b(n) \phi(z(n)) .
$$

From (3.42) and using the elementary inequality

$$
x^{\frac{1}{p}} y^{\frac{1}{q}} \leq \frac{x}{p}+\frac{y}{q}
$$

where $x \geq 0, y \geq 0$ and $\frac{1}{p}+\frac{1}{q}=1$, we observe that

$$
u(n) \leq(a(n)+b(n) \phi(z(n)))^{\frac{1}{p}}(1)^{\frac{1}{\left(\frac{p}{p-1}\right)}} \leq \frac{p-1}{p}+\frac{a(n)}{p}+\frac{b(n)}{p} \phi(z(n)) .
$$

From (3.41) and using (3.42) and (3.44), we get

$$
\begin{gathered}
\nabla^{\alpha} z(n+1)=L(n, u(n)) \\
\leq L\left(n, \frac{p-1}{p}+\frac{a(n)}{p}+\frac{b(n)}{p} \phi(z(n))\right) \\
=L\left(n, \frac{p-1}{p}+\frac{a(n)}{p}\right)+\left[L\left(n, \frac{p-1}{p}+\frac{a(n)}{p}+\frac{b(n)}{p} \phi(z(n))\right)\right.
\end{gathered}
$$




$$
\begin{gathered}
\left.-L\left(n, \frac{p-1}{p}+\frac{a(n)}{p}\right)\right] \\
\leq L\left(n, \frac{p-1}{p}+\frac{a(n)}{p}\right)+M\left(n, \frac{p-1}{p}+\frac{a(n)}{p}\right) \phi^{-1}\left(\frac{b(n)}{p} \phi(z(n))\right) \\
\leq L\left(n, \frac{p-1}{p}+\frac{a(n)}{p}\right)+M\left(n, \frac{p-1}{p}+\frac{a(n)}{p}\right) \phi^{-1}\left(\frac{b(n)}{p}\right) z(n) .
\end{gathered}
$$

Now an application of Theorem 2.1 to (3.45) yields

$$
\begin{gathered}
z(n) \leq \sum_{j=0}^{n-1} B(n-1, \alpha ; j) L\left(j, \frac{p-1}{p}+\frac{a(j)}{p}\right) \\
\prod_{k=j+1}^{n-1}\left[1+B(n-1, \alpha ; k) M\left(k, \frac{p-1}{p}+\frac{a(k)}{p}\right) \phi^{-1}\left(\frac{b(k)}{p}\right)\right] .
\end{gathered}
$$

Using (3.46) in (3.40) we get the required inequality in (3.40).

\section{Applications}

In this section we apply the fractional difference inequality established in Theorem 2.1 to obtain a bound for the solution of a fractional difference equation together with an initial condition of the form

$$
\nabla^{\alpha} u(n+1)=f(n, u(n)), u(0)=u_{0},
$$

where $f(n, r)$ be any function defined for $n \in \mathbf{N}_{0}^{+}, 0 \leq r<\infty$ and $u(n)$ : $\mathbf{N}_{0}^{+} \rightarrow \mathbf{R}$ such that

$$
\mid f(n, u(n)|\leq a(n)| u(n) \mid+b(n)
$$

for $n \in \mathbf{N}_{0}^{+}$, where $a(n), b(n)$ are as defined in Theorem 2.1. Let $u(n)$ be the solution of (4.1) for $n \in \mathbf{N}_{0}^{+}$. Using Theorem 2.1, we get

$$
|u(n)| \leq u(0) \prod_{j=0}^{n-1}[1+B(n-1, \alpha ; j) a(j)]+\sum_{j=0}^{n-1}[B(n-1, \alpha ; j) b(j)
$$




$$
\begin{aligned}
& \left.\prod_{k=j+1}^{n-1}[1+B(n-1, \alpha ; k) a(k)]\right] \\
& \leq u(0) \exp [B(n-1, \alpha ; j) a(j)]+ \\
& \sum_{j=0}^{n-1} B(n-1, \alpha ; j) b(j) \exp [B(n-1, \alpha ; k) a(k)]
\end{aligned}
$$

for $n \in \mathbf{N}_{0}^{+}$. The right hand side of the above inequality gives the bound on the solution of (4.1) in terms of the known functions.

\section{References}

[1] Agarwal, R. P. Difference equations and inequalities, Marcel Dekker, New York, (1992).

[2] Atsushi Nagai An integrable mapping with fractional difference, J. Phys. Soc. Jpn. 72, pp. 2181-2183, (2003).

[3] Deekshitulu, G. V. S. R. and Jagan Mohan, J. Fractional difference inequalities, Communications in Applied Analysis, 14, Number 1, pp. $89-98,(2010)$.

[4] Deekshitulu, G. V. S. R. and Jagan Mohan, J. Fractional difference inequalities of Bihari type, Communications in Applied Analysis, 14, Number 4, pp. 343 - 354, (2010).

[5] Deekshitulu, G. V. S. R. and Jagan Mohan, J. Fractional difference inequalities of Bihari type, Acta Et Commentationes Universitatis Tartuensis De Mathematica, 17, Number 1, pp. 19 - 30, (2013).

[6] Deekshitulu, G. V. S. R. and Jagan Mohan, J. Some New Fractional Difference Inequalities of Gronwall - Bellman Type, Mathematical Sciences, doi: 10.1186/2251-7456-6-69.

[7] Diaz, J. B. and Osler, T. J. Differences of fractional order, Math.Comp., 28, pp. 185-201, (1974).

[8] Hirota, R. Lectures on difference equations, Science-sha, (2000) (in Japanese). 
[9] Jagan Mohan, J. and Deekshitulu, G. V. S. R. Fractional Order Difference Equations, International Journal of Differential Equations, Volume 2012, Article ID 780619, 11 Pages, doi: 10.1155/2012/780619.

[10] Jagan Mohan, J. and Deekshitulu, G. V. S. R. Fractional Difference Inequalities of Gronwall Bellman Bihari Type, International Journal of Pure and Applied Mathematics, 84, Number 3, pp. 175 - 183, (2013).

[11] Lakshmikantham, V. and Leela, S. Differential and integral inequalities, Volume I, Academic Press, New York, (1969).

[12] Pachpatte, B. G. Integral and finite difference inequalities and applications,

Elsevier, The Netherlands, (2006).

[13] Podlubny, I Fractional differential equations, Academic Press, San Diego, (1999).

\section{J. Jagan Mohan}

Department of Mathematics, Birla Institute of Technology and Science Pilani, Hyderabad Campus, Hyderabad - 500078, Andhra Pradesh, India email : j.jaganmohan@hotmail.com

and

\section{G. V. S. R. Deekshitulu}

Department of Mathematics, JNTU Kakinada, Kakinada - 533003, Andhra Pradesh, India

e-mail : dixitgvsr@hotmail.com 